\title{
Acquired Methemoglobinemia
}

National Cancer Institute

\section{Source}

National Cancer Institute. Acquired Methemoglobinemia. NCI Thesaurus. Code C98805.

Methemoglobinemia that is caused by exposure to certain drugs (xylocaine and benzene) and nitrate-rich foods. 